\title{
CVD DIAMOND FILMS QUALITY CHARACTERIZATION BY ESR, SEM AND RAMAN SPECTROSCOPY
}

\author{
K. Fabisiak, J.K. PatyK and F. Rozploch
}

Institute of Physics, N. Copernicus University, Grudziądzka 5/7, 87-100 Toruń, Poland

\begin{abstract}
Polycrystalline diamond films were deposited by electron assisted hot filament chemical vapor deposition method on (001) Si substrate using a mixture of propane-butane and hydrogen as working gas. In morphology investigation of diamond films using scanning electron microscope, it was found that diamond film morphology is changing from (111), for hydrocarbon concentration below 0.5 vol.\%, via (100) to the so-called "ball-like" structure. The diamond film quality and defect structures in it were investigated by Raman and electron spin resonance spectroscopy for the purpose of obtaining basic knowledge which will aid the growth of defect free epitaxial diamond film for electronic and optical applications.
\end{abstract}

PACS numbers: $61.50 . \mathrm{Jr}$

\section{Introduction}

Diamond films were prepared by several methods such as: DC glow discharge, microwave plasma chemical vapor deposition (MP CVD), hot filament chemical vapor deposition (HF CVD), acetylene flame or plasma jet [1-5]. Growth rate as well as qualities of diamonds produced are not very sensitive to the kind of the hydrocarbon source but are strongly influenced by deposition parameters such as: substrate temperature, total pressure in the reaction chamber, total flow rate of the working gas and hydrocarbon concentration.

The diamond films are expected to find an application as heat sink in electronic devices, protective films, transistors operating at high temperatures, optical and microwa ve windows [6]. However, the diamond films grow epitaxially only on diamond substrates and not on other kinds of substrates for a variety of reasons such as strain in the films and formation of $s p^{2}$ carbons, etc.

Raman spectroscopy is a powerful tool in characterizing carbon material. A single Raman peak is observed at $1332.5 \mathrm{~cm}^{-1}$ for diamond and at $1587 \mathrm{~cm}^{-1}$ for graphite. So far, Raman spectroscopy has been mainly used to confirm the existence of diamond phase in the deposit. We focused our efforts on deep diamond Raman line analysis. The ESR results fully supported the interpretation of the Raman spectrum broadening. 


\section{Sample preparation}

The diamond films were deposited on (001) Si substrates by EA HF CVD (electron assisted HF CVD) method using a mixture of propane-butane and hydrogen as a working gas. The experimental setup was described in the recent paper. The gas pressure was 80 torr and the flow rate was $100 \mathrm{sccm}$ (standard cubic centimeter per minute). The substrate temperature was measured by thermocouple and kept at $850^{\circ} \mathrm{C}$. The substrate was placed about $10 \mathrm{~mm}$ below tungsten filament heated to $2300^{\circ} \mathrm{C}$.

In order to increase the nucleation density of diamonds, Si wafers were polished with diamond paste of $1 \mu \mathrm{m}$ corn diameter size, washed with distilled water and, then, alcohol using ultrasonic cleaner and finally dried in the air.

\section{Results and discussion}

The morphology of the deposited diamond films was observed to depend not only on deposition parameters but also on the substrate surface preparation. It was observed that it is very difficult to deposit continuous diamond film on unpolished substrates. As one can see in Fig. 1A, the diamond nucleation takes place on surface defects where nucleation density is a few order of magnitude higher in comparison to the places free of mechanical defects.
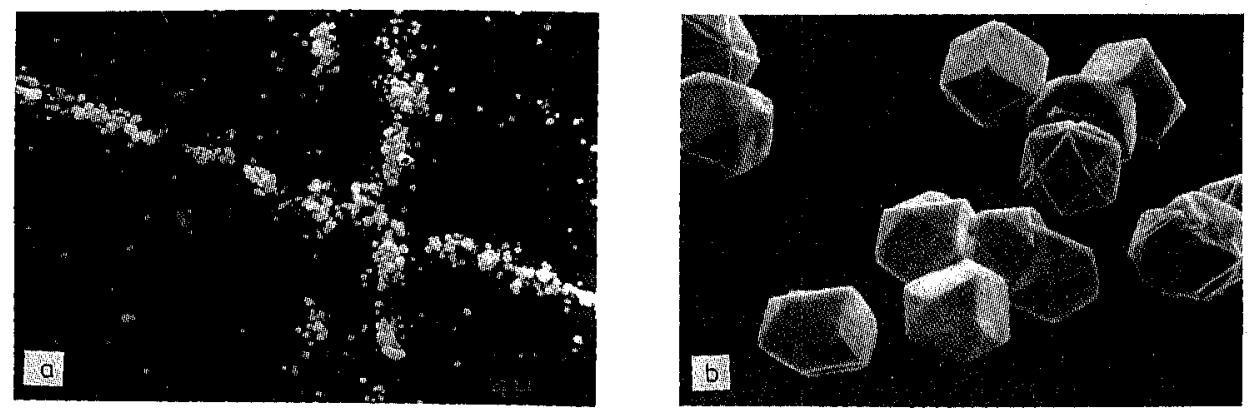

Fig. 1. (A) Beginning of the diamond microcrystals nucleation on surface defects. (B) Different structures of diamond crystallites.

It confirms the idea that an enhanced surface adsorption of free radicals, responsible for solid phase growth, can take place on surface defects.

If the substrate surface is free of mechanical defects only separate diamond microcrystals were deposited as is shown in Fig. 1B.

As one can see in Fig. 1B, diamond microcrystals can have different structures such as cubo-octahedron twinned cubo-octahedron, decahedral-Wulff-polyhedron and icosahedron, and all the forms are deposited under exactly the same conditions.

Unpolished (001) Si surface is homogeneous and has the same surface adsorption capability in all its points. The only reasonable explanation of the deposition, in almost the same time and almost in the same place on the substrate, of diamond 
microcrystals having different structure is their nucleation in the gas phase and not an substrate surface.

The precursors for diamond nuclei are probably cage compounds of carbon and hydrogen atoms. The smallest compound that has the skeleton of diamond structure is adamantane. The precursors for D-Wulff-polyhedron and icosahedron are probably bicyclo-octane or tetracyclo-dodecane. The problem with this hypothesis is that these compounds are not stable above $500^{\circ} \mathrm{C}$ [7]. However, they can probably grow to diamond nuclei under supersaturation of hydrocarbon radicals and atomic hydrogen.

Other possibility is that the precursors for diamond microcrystals having fivefold symmetry can be fullerenes $\mathrm{C}_{60}$ and $\mathrm{C}_{70}$, as it was already proposed in our recent paper [8]. According to our experiments, diamonds indeed nucleate with high nucleation density on unpolished Si substrate covered by fullerenes. It should be noticed that up to now the problem of diamond nucleation is still unsolved.

If the substrate surface is polished with diamond paste before deposition it is relatively easy to obtain continuous diamond films having different morphology (see Fig. 2B) depending on the working gas composition. In general, the morphology of the diamond films is changing in direction shown in Fig. 2C.

Diamond film quality measure is given by their Raman line half width. The examples of the Raman spectrum of our diamond films is shown in Fig. 2A. This figure shows also the decomposition of each Raman spectrum in order to show the contribution of the pure diamond phase, $s p^{2}$ carbon phase ( $D$ and $G$ lines) and photoluminescence background to our experimental spectra. Such decomposition allows an estimation of the intensity ratio of $D$ to $G$ lines, which is the indication of the $s p^{2}$ cluster size. The results of the Raman spectroscopy measurement are collected in Table.

With increasing hydrocarbon concentration several trends were observed:

a) broadening of the diamond Raman line,

b) shift of the diamond Raman line to higher wave numbers,

c) decrease in ratio of intensities of the line $D$ to $G\left(I_{D} / I_{G}\right)$.

The broadening of the diamond Raman line indicates that diamond film quality is decreasing which can be caused by $s p^{2}$ phase admixture.

The increase of the $s p^{2}$ clusters' size is indicated by a decreasing $I_{D} / I_{G}$ ratio. The $s p^{2}$ carbon phase dispersed in the diamond film in general should create interval stresses, because of different $\mathrm{C}-\mathrm{C}$ bond length. The stresses are responsible for the observed diamond Raman line shift from its $1332.5 \mathrm{~cm}^{-1}$ ideal position. The internal stresses can be estimated using the theory developed by Fitzer and Rozploch [9]. The results are listed in Table.

The above ideas are fully supported by ESR measurements. As one can see from Table, the paramagnetic center concentration increases when the quality of the diamond film becomes worse (in sense of a decrease in ratio $I_{D} / I_{G}$ ).

Additionally, the ratio of spin concentration measured at liquid nitrogen temperature $N_{\mathrm{LN}}$, and room temperature $N_{\mathrm{RT}}$ decreases from 3.6 to 2.2 , indicating that contribution of non-localized spins increases. This can be associated with an increase in the $s p^{2}$ cluster size. Finally, we can conclude that both Raman and ESR results correlate well with morphological changes of our diamond films. 

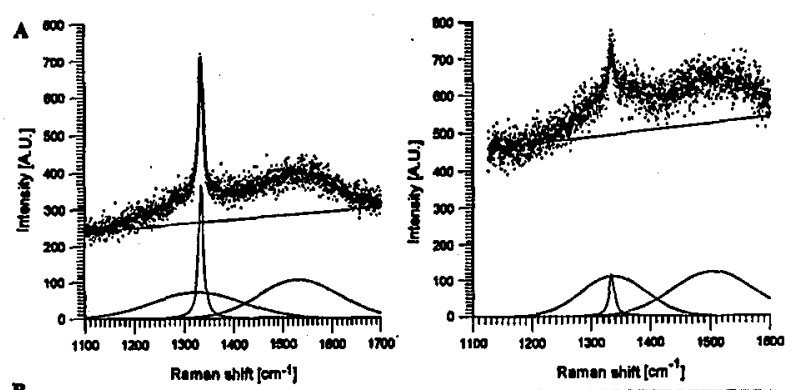

B

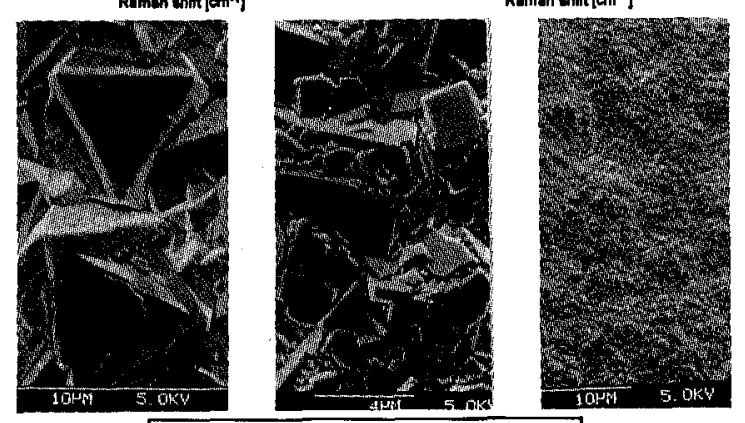

C

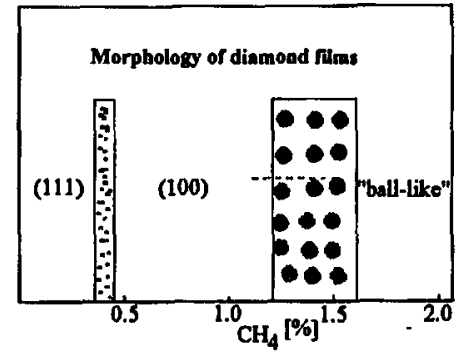

Fig. 2. (A) Experimental Raman spectra and their numerical decomposition; (B) different morphologies of diamond films; (C) diagram of diamond films morphology (shadowed regions indicate mixed morphology).

TABLE

The results of Raman and ESR spectroscopy.

\begin{tabular}{c|c|c|c|c|c|c|c}
\hline \hline \multirow{2}{*}{$\begin{array}{c}\text { Hydrocarbon } \\
\text { concentration } \\
\text { [vol.\%] }\end{array}$} & \multicolumn{3}{|c|}{ Raman spectroscopy } & \multicolumn{4}{c}{ ESR } \\
\cline { 2 - 3 } & $\begin{array}{c}\text { Diamond line } \\
\text { Peak position } \\
{\left[\mathrm{cm}^{-1}\right]}\end{array}$ & $\begin{array}{c}\Delta \omega_{1 / 2} \\
{\left[\mathrm{~cm}^{-1}\right]}\end{array}$ & $I_{D} / I_{G}$ & $\begin{array}{c}\sigma \\
{[\mathrm{GPa}]}\end{array}$ & $\begin{array}{c}\Delta B_{\mathrm{pp}} \\
{[\mathrm{mT}]}\end{array}$ & $\begin{array}{c}N_{\mathrm{RT}} \\
\times 10^{18} \\
{\left[\mathrm{~cm}^{-3}\right]}\end{array}$ & $N_{\mathrm{LN}} / N_{\mathrm{RT}}$ \\
\hline 0.15 & 1332.2 & 9.3 & 0.65 & 0.0394 & 0.17 & 0.6 & 3.60 \\
0.30 & 1332.6 & 9.6 & 0.56 & 0.1180 & 0.20 & 0.8 & 3.50 \\
0.50 & 1333.5 & 11.6 & 0.52 & 0.4330 & 0.28 & 1.4 & 3.45 \\
1.00 & 1334.2 & 13.5 & 0.45 & 0.8265 & 0.33 & 2.4 & 3.20 \\
1.50 & 1335.8 & 15.6 & 0.41 & 1.2860 & 0.38 & 7.7 & 2.20
\end{tabular}




\section{Acknowledgments}

This work is financially supported by the State Committee for Scientific Research (Republic of Poland) grant No. 223069203.

\section{References}

[1] M. Kamo, Y. Sato, S. Matsumoto, N. Setaka, J. Cryst. Growth 62, 642 (1983).

[2] S. Matsumoto, J. Mater. Sci. Lett. 4, 600 (1985).

[3] Y. Hirose, S. Ananuma, N. Okada, K. Komaki, in: Proc. 1st Int. Symp., Vol. 89, Electrochem. Soc., Pennington (NJ) 1989, p. 80.

[4] G. Jansen, W.J.P. van Enckefort, J.J.D. Schaminee, W. Vollenberg, L.J. Giling, M. Seal, J. Cryst. Growth 104, 752 (1990).

[5] M. Kamo, Y. Sato, S. Matsumoto, N. Setaka, Jpn. J. Appl. Phys. 21, L183 (1982).

[6] K. Shenai, R.S. Scott, B.J. Baliga, IEEE Trans. Electron. Devices 36, 1811 (1989).

[7] S. Matsumoto, unpublished data (1978).

[8] W. Marciniak, K. Fabisiak, S. Orzeszko, F. Rozpłoch, J. Cryst. Growth 123, 587 (1992).

[9] E. Fitzer, F. Rozpłoch, High Temp.-High Press. 20, 449 (1988). 